BIOMEDICA

Vol. 6. Nos. 3 y 4 - 1986

\title{
DENGUE EN COLOMBIA 1/
}

\author{
JORGE BOSHELL* , HERNANDO GROOT*, MANUEL G. GACHARNA**, GLADYS MARQUEZ* , MARTHA GONZALEZ*, \\ MARTHA O. GAITAN*, CHRISTINE BERLIE*, MANCEL MARTINEZ***
}

\begin{abstract}
El Gobierno Colombiano estableció una campaña que erradicó el Aedes aegypti de su territorio en atención a las recomendaciones que hizo la Oficina Sanitaria Panamericana en 1947. Esta campaña consiguó desaparecer el dengue endémico durante aproximadamente 20 años, apareciendo de nuevo en forma explosiva con la epidemia de dengue 2 en la Costa Atlántica (1971-1972), seguida de dos epidemias bien documentadas de dengue 3 (1975-1977) y dengue 1 en 1978. Se hace un resumen de las actividades que desarrolla el Laboratorio de Virología del Instituto Nacional de Salud para apoyar el diagnóstico de esta enfermedad en el país incluyendo el primer aislamiento de dengue 4 en 1982, la actividad de los virus dengue 1, 2 y 4 detectada hasta la fecha, los hallazgos clínicos y virológicos en un caso fatal de enfermedad hemorrágica asociada a infección por virus del dengue y un breve recuento de la epidemia de Tumaco en la Costa Pacífica en la cual se comprobó actividad simultánea de dengue 1 y 2 . Finalmente se informa sobre el estado de infestación que tiene el país actualmente con el Aedes aegypti y sobre la actividad del virus de fiebre amarilla en focos selváticos vecinos a ciudades altamente infestadas, detectada en el mes de enero de 1987 en Colombia.
\end{abstract}

\section{ANTECEDENTES}

La organización que estableció el gobierno colombiano para erradicar el Aedes aegypti de su territorio, siguiendo las recomendaciones del Consejo Directivo de la Oficina Sanitaria Panamericana (OSP), reunido en Buenos Aires en septiembre de 1947, se canalizó en su comienzo a través del Servicio Anti-aegypti del Ministerio de Salud. Este servicio inició trabajos en agosto de 1950, contó con la asesoría permanente de la OSP hasta su culminación y después de 10 años logró cumplir con las formalidades exigidas por la misma oficina para declarar el país libre del mosquito (1). La tabla 1 resume algunas de las actividades que se desarrollaron, las cuales tuvieron un costo inferior a US $\$ 400.000$ (2). El $28 \%$ del territorio se encontraba infestado y comprendía las poblaciones situadas entre las costas y la cordillera oriental ya que el transmisor nunca había sido encontrado en los territorios situados al oriente de la misma. Al finalizar la década el Consejo Directivo de la Oficina Sanitaria Panamericana declara el territorio colombiano libre de Aedes aegypti y recomienda la necesidad de "mantener una estricta vigilancia del mosquito en puertos marítimos, aéreos y terrestres mientras existiera área infestada en el continente" (2). La ciudad limítrofe de Cúcuta en nuestra frontera con Venezuela requirió tratamientos especiales, entre otras razones por tener una cepa de Aedes resistente al DDT y para su control definitivo fue necesario invertir la sexta parte del presupuesto general. El objeto fundamental de todo este esfuerzo era evitar el riesgo urbano continental de la fiebre amarilla

\footnotetext{
* Grupo de Virología INS.

** Dirección Epidemiología Minsalud.

***Vigilancia Epidemiológica INS.
}

1/Trabajo presentado en la Conferencia Internacional sobre Dengue que tuvo lugar en Mérida, Yucatán, México, en febrero 18-20 de 1987. auspiciada por la Organización Mundial de la Salud (OPS) y el International Development Research Centre (IDRC). de Canadá. Es reproducido con autorización de estas entidades 
TABLA 1

ALGUNAS ACTIVIDADES DESARROLLADAS
POR EL SERVICIO ANTI-AEGYPTI
COLOMBIA, $1950-1960$ *

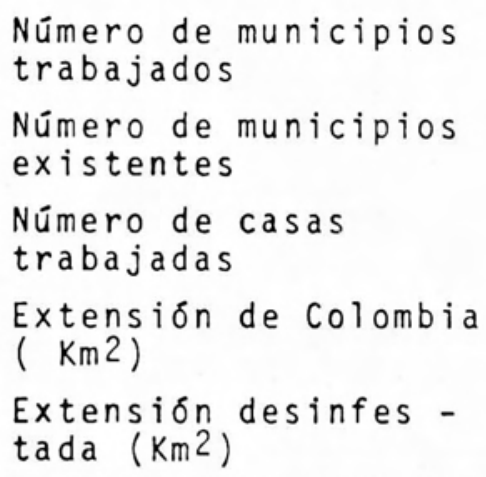

* Datos tomados del Informe final del Programa de Erradicación del Aedes aegypti de Colombia.

pero consiguió también desaparecer el dengue del panorama nacional; esta enfermedad era endémica en el país por lo menos hasta el año $1952(3,4)$ y con la desaparición del mosquito transmisor se extinguió durante aproximadamente 20 años no solo de las consultas clínicas sino también de la cátedra de las escuelas de Medicina y Salud Pública.

\section{LAS EPIDEMIAS}

La enfermedad reapareció por primera vez en forma explosiva con la epidemia de dengue 2 que experimentó la Costa Atlantica colombiana en la segunda mitad de 1971 afectando a una población cercana a las 500.000 personas $(4,5,6,7)$. Es reconocida la tenacidad que tiene el Aedes aegypti para invadir nuevos territorios y en Colombia fue posible seguir su rastro por la aparición de epidemias en las poblaciones recientemente infestadas en el interior del país. Los detalles cronológicos relativos a esta reinvasión han sido publicados anteriormente (7).

Vinieron luego, en 1975, los primeros brotes de dengue 3 que en el interior del país afectaron a 200.000 personas aproximadamente. Sin embargo, teniendo en cuenta las tasas de ataque en algunas poblaciones del Valle del río Magdalena en donde las epidemias fueron particularmente bien estudiadas y considerando la magnitud con que se presentaron, globalmente debieron afectar a una población superior a los 400.000 (9). Solamente en Antioquia se estiman 100.000 casos en una epidemia que inició su curso en la segunda mitad de 1977 y terminó en octubre de 1978 (10).

El Dengue es de notificación obligatoria en Colombia a partir de 1978. Este hecho permitió registrar la tercera gran epidemia con los casos clínicos informados a la Dirección de Epidemiología del Ministerio de Salud (Figura 1), comenzó en diciembre de 1977 (7), se diseminó por el territorio durante los dos años siguientes y llegó a su punto máximo en noviembre de 1978 dibujando una curva epidémica casi simétrica. Sólo se informaron 28.078 casos clínicos oficialmente (11) (tabla 2), pero es bien sabido que uno de los imponderables en el registro estadístico de esta enfermedad es el fenómeno del subregistro, debido a que una vez identificada la epidemia de Dengue, los enfermos mismos reconocen su condición y evitan razonablemente la consulta médica a menos que presenten alguna complicación. Teniendo en cuenta las experiencias anteriores se estima en 770.000 casos la magnitud de este brote (7).

El dengue 4 se aisló por primera vez en julio de 1982 a partir del suero de un enfermo residente en Valledupar, Cesar, ciudad de la Costa Atlántica por la cual estaba pasando una onda epidémica en ese momento. La transmisión de este serotipo produjo brotes con manifestaciones clínicas discretas cuya magnitud real nunca fue posible medir; su actividad se registró en todo el territorio hasta enero de 1984 fecha en que se aisló por última vez de un suero remitido por el Servicio de Salud de Risaralda, en el interior del país. Es de suponer que la mayoría de casos clínicos presentados entre 1982 y 1984 fueron causados por este virus teniendo en cuenta que los otros tres serotipos habían circulado con anterioridad, sin embargo, debe tenerse en cuenta que en ese mismo tiempo se registró también transmisión de dengue 1 y 2 (tabla 2). La transmisión del dengue fue particularmente activa en 1983 y el registro de casos sospechosos en lạ Dirección de Epidemiología del Ministerio de Salud llegó a cifras similares a las de 1978-79 cuando la gran epidemia de DEN-I (tabla 2). Este 
DENGUE EN COLOMBIA

FIGURA 1

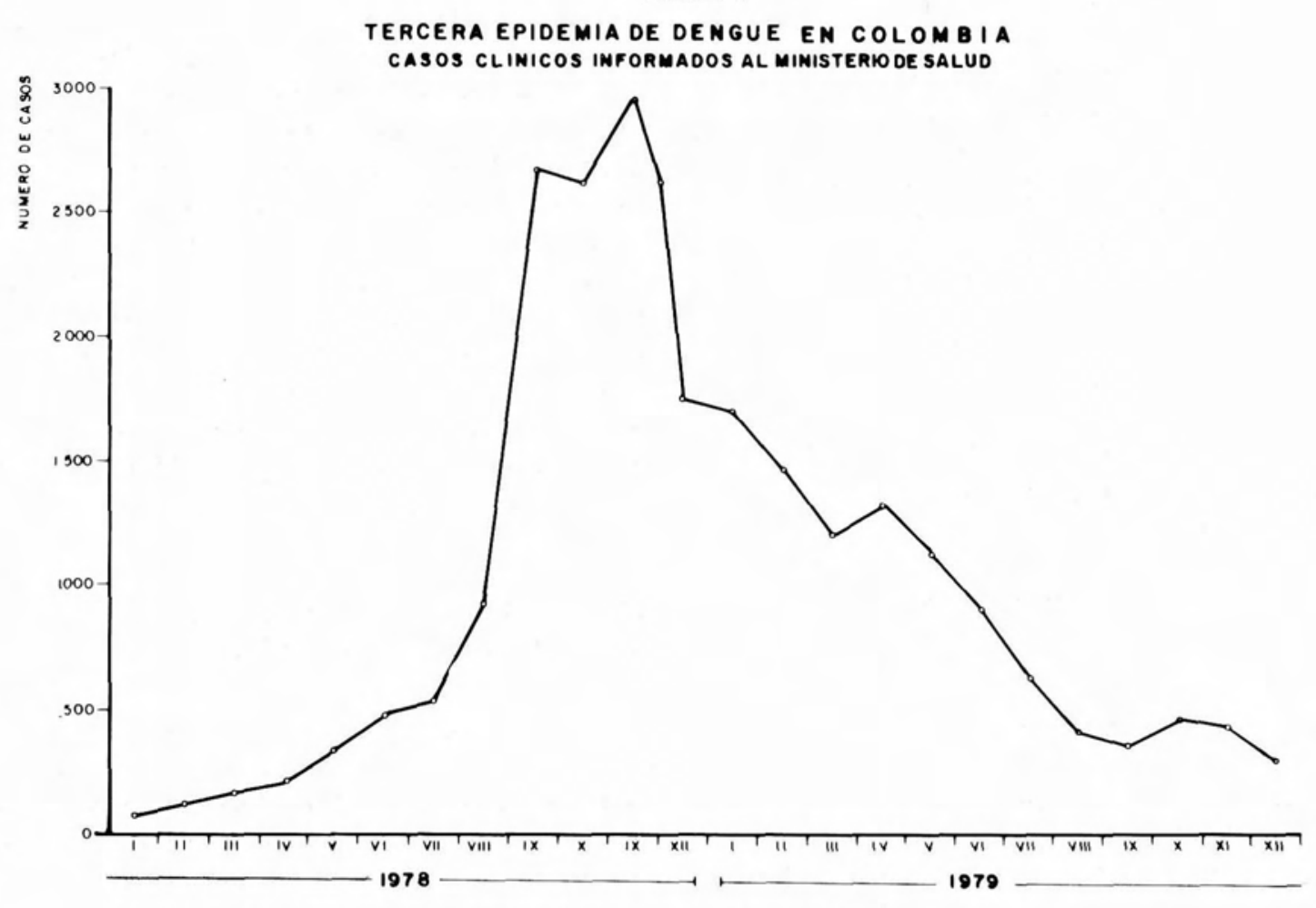

FUENTE DiReccion de EPIOEMIOL OGIA, MINISTERIO DE SALUD PUBLICA DE COL OMBIA

TABLA 2

DENGUE EN COLOMBIA

CASOS CLINICOS INFORMADOS AL MINISTERIO DE SALUD (1) EN RELACION CON SEROTIPOS AISLADOS.

\begin{tabular}{|c|c|c|}
\hline AÑO & $\begin{array}{l}\text { NÜMERO } \\
\text { CASOS }\end{array}$ & $\begin{array}{l}\text { SEROTIPOS } \\
\text { AISLADOS (2) }\end{array}$ \\
\hline 1978 & 15944 & D1. \\
\hline 1979 & 12134 & D1. \\
\hline 1980 & 8984 & $\mathrm{D} 1, \mathrm{D} 2$ \\
\hline 1981 & 7790 & D1. \\
\hline 1982 & 6790 & $\mathrm{D} 1, \mathrm{D} 2, \mathrm{D} 4$ \\
\hline 1983 & 14081 & $\mathrm{D} 1, \mathrm{D} 2, \mathrm{D} 4$ \\
\hline 1984 & 7540 & $\mathrm{D} 1, \mathrm{D} 4$ \\
\hline 1985 & 7797 & $\mathrm{D} 1, \mathrm{D} 2$ \\
\hline $1986(3)$ & 5576 & $\mathrm{D} 1, \mathrm{D} 2$ \\
\hline
\end{tabular}

(1) El Dengue es una enfermedad de notificacion obligatoria a la Dirección de Epidemiología, Ministerio de Salud a partir de 1978.

(2) Grupo de Virología, Instituto Nacional de Salud, D1, D2 y $D 4=$ Dengue 1,2 y 4 respectivamente.

(3) Información aún no consolidada (parcial). hecho correlaciona bieh con una mayor actividad de laboratorio en la cual se aislaron 18 cepas de virus (tabla 3). Las cepas de DEN-1 fueron aisladas en el Laboratorio de Virus de la Universidad del Valle y remitidas a Bogotá para su identificación; se obtuvieron durante una epidemia registrada en Palmira, Valle (213.433 habitantes) ciudad que experimentaba por primera vez una epidemia de Dengue. Este fue el segundo año en que se registró actividad persistente de tres serotipos simultáneamente. Destaca el hecho que el DEN-3 no se ha confirmado en circulación simultánea con los otros virus y que su transmisión no se registra desde la epidemia que ocasionó 10 años atrás.

El ultimo brote de dengue que se ha presentado con cáracter de epidemia ocurrió en Tumaco, Nariño, entre octubre de 1985 y marzo de 1986 . Es el puerto septentrional que tiene Colombia en la Costa Pacífica, cerca de la frontera con Ecuador y registró transmisión simultánea de DEN-1 y 2. (12). Desde entonces se ha registrado transmisión de DEN-1 en Cundinamarca y Tolima. 
TABLA 3

INSTITUTO NACIONAL DE SALUD

DIAGNOSTICO DE DENGUE

LABORATORIO DE VIROLOGFA

\begin{tabular}{|c|c|c|c|c|c|c|c|}
\hline \multirow{3}{*}{$\begin{array}{l}\text { AN̂̃o } \\
1978\end{array}$} & \multicolumn{6}{|c|}{ Aislamientos (AV) VIRUS DENGUE (1) } & \multirow{2}{*}{$H I(\mp)(2)$} \\
\hline & \multirow{2}{*}{$\frac{D 1}{\text { (3) } 1}$} & \multirow{2}{*}{$\frac{\mathrm{D} 2}{0}$} & \multirow{2}{*}{$\frac{D 3}{0}$} & \multirow{2}{*}{$\begin{array}{c}D 4 \\
0\end{array}$} & \multicolumn{2}{|c|}{ A/V/ INTENTOS A/V } & \\
\hline & & & & & (4) $1 / 62$ & $(10)$ & $12 / 15 \quad(86)$ \\
\hline 1979 & 4 & 0 & 0 & 0 & $4 / 28$ & (14) & $21 / 25 \quad(84)$ \\
\hline 1980 & 2 & 2 & 0 & 0 & $4 / 64$ & ( 6$)$ & $10 / 15 \quad(67)$ \\
\hline 1981 & 4 & 0 & 0 & 0 & $4 / 50$ & ( 8$)$ & $7 / 26 \quad(27)$ \\
\hline 1982 & 1 & 1 & 0 & 2 & $4 / 73$ & ( 5$)$ & $16 / 19 \quad(84)$ \\
\hline 1983 & 9 & 3 & 0 & 6 & $18 / 107$ & (17) & $(108)$ \\
\hline 1984 & 5 & 0 & 0 & 3 & $8 / 19$ & (42) & $6 / 10 \quad(60)$ \\
\hline 1985 & 3 & 3 & 0 & 0 & $6 / 136$ & $(4)$ & $(86)$ \\
\hline 1986 & 7 & 6 & 0 & 0 & $13 / 44$ & (29) & $38 / 44 \quad(86)$ \\
\hline TOTALES & 42 & 15 & 0 & 11 & $67 / 483$ & (14) & $125 / 171(73)$ \\
\hline
\end{tabular}

(1) $01,02,03,04 .-$ Virus Dengue $1,2,3$ y 4 respectivamente.

(2) $\mathrm{HI}=$ Inhibición de la Hemaglutinación en sueros pareados:

(3) Se aistaronversiones / Total de sueros pares.

(3) Se aislaron 7 cepas de virus durante la epidemia pero sol lamente una
se identificó con fijación de Complemento y Neutralización.

(4) $\left(x^{\prime}\right)$

\section{DENGUE HEMORRAGICO}

El cuadro clínico del dengue colombiano ha sido hasta la fecha el de un dengue clásico, con manifestaciones hemorrágicas de frecuencia e intensidad variables tales como han sido descritas en los textos (13, 14, 15). Desde la primera epidemia de DEN-2, la introducción de cada serotipo nuevo creó simpre la expectativa del Dengue Hemorrágico-Dengue Hemorrágico con Shock (DH-DSS) pero hasta la fecha sigue siendo un fantasma en el país. En un caso intrigante, posiblemente el único registrado hasta la fecha en Colombia de síndrome febril con hemorragia masiva que hemos podido asociar a una infección aguda por virus del dengue, no fue posible reunir los criterios de la OMS para hacer diagnóstico de DH-DSS y presentó en cambio un síndrome hemolítico muy severo (16). Era un estudiante del ejército colombiano, de sexo masculino, de 19 años de edad, quien durante sus prácticas de campo inició súbitamente una enfermedad febril con diarrea y malestar que en poco tiempo comprometió gravemente su estado general. Después de una corta remisión de síntomas a los 8 días de enfermedad, comenzó a presentar intenso dolor lumbar asociado a fiebre, coluria e ictericia con hemorragias subcutáneas y de orofaringe; además dolor abdominal difuso con hepatoesplenomegalia. Fue remitido al Hospital Militar Central de Bogotá y falleció 10 horas más tarde. El cuadro hemático de su ingreso mostraba leucocitosis $(29000 / \mathrm{ml})$, hemoglobina baja $(5.0 \mathrm{gm} /$ $\mathrm{dl})$, hematocrito bajo $(8 \%)$ y los hallazgos más llamativos de la química sanguínea fueron la evidencia de hemólisis (Bilirrubina Total $9.0 \mathrm{mg} / \mathrm{dl}$, Bilirrubina Directa $7.0 \mathrm{mg} / \mathrm{dl}$ ). En la necropsia se evidenció hemorragia visceral generalizada con eritrofagocitosis. La prueba de Inhibición de la Hemaglutinación (HI) mostró una reacción de tipo secundario con los 4 antígenos de dengue y fiebre amarilla e IgM-ELISA con los 4 antígenos de dengue dió reacción a título alto. Los intentos de aislamiento de virus fueron negativos en células C6/36 y en la inoculación de Toxorhinchytes amboinensis. Seis compañeros de este paciente que presentaron un síndrome febril compatible con dengue mostraron una respuesta en la prueba $\mathrm{HI}$ idéntica. Un año antes de este episodio se había aislado DEN-1 en el área.

\section{BROTE EPIDEMICO DE FIEBRE AMARILLA SELVATICA EN SANTANDER}

La actividad de la fiebre amarilla (FA) está repuntando estos días en las selvas del departamento de Santander que bordean el río Magdalena Medio en su margen derecha. Hasta la fecha 9 de los 10 casos de FA selvática confirmados en el curso de este año han sido producidos en esa zona, cuatro de los cuales han fallecido (tabla 4). Todos los casos han sido diagnosticados serológicamente detectando inmunoglobulina M (IgM) antiamarílica por ELISA excepto el caso No. 5 del cual no se obtuvo muestra de suero. Los tres

TABLA 4

INSTITUTO NACIONAL. DE SALUD CASOS DE FIEBRE AMARILLA, 1987

LABORATORIO DE VIROLOGIA

\begin{tabular}{|c|c|c|c|c|c|}
\hline No. & FECHA & $\begin{array}{l}\text { REGISTRO } \\
\text { VIROLOGIA }\end{array}$ & $I G M-F A$ & $\begin{array}{l}\text { Histopato- } \\
\text { LOGIA. }\end{array}$ & Procedencia \\
\hline 1 & $1-7-87^{\circ}$ & 349831 & $1: 800$ & Pasitiva & CIMITARRA, SANTANDER \\
\hline 2 & $1-20-87$ & $\begin{array}{l}349854 \\
349919 \\
349929\end{array}$ & $\begin{array}{l}>1: 200 \\
1: 12800 \\
1: 12800\end{array}$ & $\begin{array}{l}57351 \\
\text { VIVE }\end{array}$ & CIMITARRA, SANTANDER \\
\hline 3 & $1-20-87$ & $\begin{array}{l}349856 \\
349920 \\
349930\end{array}$ & $\begin{array}{c}1: 200 \\
1: 51200 \\
>1: 51200\end{array}$ & VIVE & CIMITARRA, SANTANDER \\
\hline 4 & $1-20-87$ & 349921 & $1: 1600$ & NO HUBO & Mapiripan, Meta \\
\hline 5 & $1-27-87$ & NO & NO & $\begin{array}{l}\text { Positivo } \\
57353\end{array}$ & CIMITARRA, SANTANDER \\
\hline 6 & $11-5-87^{\circ}$ & 349933 & $1: 6400$ & VIVE & CIMITARRA, SANTANDER \\
\hline 7 & $11-10-87$ & 349952 & $>1: 200$ & $\begin{array}{l}\text { Positivo } \\
57356\end{array}$ & BETULIA, SANTANDER \\
\hline 8 & $11-10-87$ & 349953 & $>1: 200$ & VIVE & Zapatoca, SANTANDER \\
\hline 9 & $11-12-87$ & 350001 & $>1: 200$ & VIVE & LANDAZURI， SANTANDER \\
\hline 10 & $11-12-87$ & 350002 & $>1: 200$ & $\begin{array}{l}\text { Positivo } \\
57357\end{array}$ & BOLIVAR, SANTANDER \\
\hline
\end{tabular}

- Infectados simultasneamente. 
casos fatales restantes $(1,4,6$ y 7$)$ mantuvieron confirmación histopatológica adicional y el caso procedente del departamento del Meta fue diagnosticado por serología únicamente.

\section{SITUACION ACTUAL DEL AEDES}

A finales de 1969 los esfuerzos que se hicieron para controlar el $A$. aegypti fueron insuficientes y la infestación que se registró dos años más tarde comprometía el norte del país hasta el litoral Atlántico (7); en 1981 se encontraron larvas en una localidad de la comisaría del Vaupés situada en el extremo oriental selvático del país y en el territorio que originalmente había sido desinfestado (17). Aunque este mosquito sigue siendo de hábitos domésticos y se encuentra preferencialmente en áreas urbanas de Colombia, existen registros de alta infestación tanto de adultos como de larvas en casas aisladas de zonas rurales cuya carretera de acceso más próxima se encuentra a $2 \mathrm{kms}$. de distancia (18). La distribución vertical ha llegado hasta los $2200 \mathrm{~m}$. sobre el nivel del mar en el municipio de Málaga, Santander, en donde se encontraron más del $12 \%$ de casas con larvas (19).

Actualmente se estima una infestación de 330.000 $\mathrm{km}^{2}$ que están por debajo de $1500 \mathrm{~m}$. sobre el nivel del mar, con 3 millones de viviendas y una población de 15 millones de habitantes (20).

Los informes relacionados con la aparición del $\mathrm{Ae}$ des albopictus en algunos países de América han despertado inquietud en la Sección de Aedes del Ministerio de Salud la cual ha obtenido financiación internacional para iniciar su pesquisa (20).

Teniendo en cuenta el estado de infestación del país por Aedes aegypti el Ministerio de Salud organizó una red de vigilancia de la fiebre amarilla y el dengue. El propósito de esta red es identificar con rapidez la actividad de estos virus para iniciar medidas de control oportunas. El trabajo de laboratorio se ha facilitado enormemente con las técnicas enzimáticas que permiten hacer diagnóstico rápido de estas dos enfermedades y su utilidad práctica quedó demostrada en el país con el diagnóstico inmediato que se hizo de la situación de fiebre amarilla en Santander, el cual ha permitido tomar medidas oportunas para evitar un brote de mayores proporciones.

\section{SUMMARY}

In 1947 the Colombian goverment established a campain for the erradication of Aedes aegypti in the country, following the recomendations of the Pan American Sanitary Bureau. This campaign caused the disappearance of endemic dengue for approximately 20 years until it reappeared in explosive form with the epidemic of dengue 2 on the Atlantic Coast (1971-1972), followed by two documented large epidemics of dengue 3 (1975-1977) and dengue 1 in 1978. A summary is given of the activities developed by the virus laboratory of the National Institute of Health to aid the diagnosis of this disease in the country, including the first isolation of dengue 4 virus in 1982, the activity of dengue 1,2 and 4 viruses to the present time, the clinical and virological findings of a hemorragic case associated to dengue virus infection and a short report of the epidemic of Tumaco on the Pacific Coast, in which the simultaneous activity of dengue 1 and dengue 2 viruses was demonstrated. Finally, information is given on the current status of Aedes aegypti infestation in the country and about the activity of yellow fever virus in sylvatic foci close to heavily infected cities, detected in the month of January, 1987 in Colombia.

\section{AGRADECIMIENTOS}

La colaboración de los Servicios Seccionales de Salud del país ha sido definitiva para la realización de la Vigilancia Epidemiológica.

El doctor Duane J. Gubler, Director del laboratorio de Centers For Disease Control, San Juan, Puerto Rico, confirmó algunos resultados de nuestra prueba IgM-ELISA para Dengue.

La señora Alcira Díaz gentilmente colaboró con la transcripción del texto.

\section{BIBLIOGRAFIA}

1. OPS. Guía de los informes de la Campaña de Erradicación del Aedes aegypti en las Américas. Washington: Organización Parnamericana de la Salud, 1960; Publicaciones Varias, No. 49, 8-10.

2. Programa de Erradicación del Aedes aegypti, Ministerio de Salud Pública de la Repúbtica de Colombia, Informe final, 1961. 
3. Groot $\mathrm{H}$. Estudios sobre virus transmitidos por artrópodos en Colombia. Rev Acad Colomb Cienc Exac Fisic Nat 1964; 12:2-23.

4. Groot H. Dengue Fever: present and potential problems in Colombia. Industry and Tropical Health: VIII. Boston: Harvard University. Sin fecha.

5. Sanmartin C. Experiencias epidemiológicas en subpaíses superdesarrollados. Traducción de la Francis Craig Lecture para ASTMH. Ant Med, 1974; 24(2): 103-109.

6. Sanmartin C. Dengue Symposium, March 1972. Port of Spain, Trinidad.

7. Groot H. The reinvasion of Colombia by Aedes aegypti: Aspects to remember. Second Soper Lecture. Am J Trop Meủ Hyg 1980; 29(3): 330-338.

8. Groot $\mathrm{H}$, et al. Recent outbreaks of dengue in Colombia: Pan American Health Organization; 1979; Sci Publ No. 375, 3139.

9. Groot H. Comunicación personal.

10. Jaramillo C, Hoyos H, Builes A, Agudelo L, Olivares P. de Restrepo, B de los Rios, J Acta Med Col, 1979; 4(3): 119-133.

11. Ministerio de Salud Pública de Colombia, Dirección de Epidemiología. 1986.

12. Boshell J, Gaitán MO, Tenganá H, Gacharná MG, Rey M. Epidemia de Dengue en Tumaco, 1985. Evidencia de transmisión simultánea de los serotipos 1 y 2. En preparación.
13. EhrenKrantz NJ, Venture AK, Cuadrado RR, Pond WL, Porter JE. Pandemic dengue in Caribbean countries and the Southern United States. Past, present and potential problems. N Engl J Med, 1971; 285: 2460-2469.

14. Groot H. y Boshell J. Dengue hemorrágico y fiebre amarilla. En: Chalem F, Escandón J, Campos J, Esguerra R. Medicina Interna. Cali: Editorial Norma, 1986.

15. Guide for Diagnosis, Treatment and Control of Dengue Haemorrhagic Fever, Second Edition, Geneve: World Health Organization, 1980.

16. Espinal CA, Boshell SJ, Narváez C, Carmona F, Berlie Ch, Gaitán MO, Buitrago B, Ossa LJ. y Calderón H. Síndrome hemolítico-hemorrágico asociado a una infección aguda por virus dengue.

17. Suárez MF, Nelson MJ, Morales A, Archila L, Galvis E, Guzmán J, Dussan R. Aedes aegypti en áreas rurales de Colombia. Reunión sobre Aedes aegypti, Ministerio de Salud y OPS, marzo 6, 1984.

18. Morales A. Aedes aegypti en zona rural del Municipio de la Mesa (Cundinamarca), Colombia, S.A., Biomédica 1981; 1(4): 223.

19. Suárez MF, y Nelson MJ. Registro de Altitud del Aedes aegypti en Colombia, Biomédica 1981; 1(4): 225.

20. Velandia V. Jefe Sección de Aedes, Dirección de Campañas Directas del Ministerio de Salud. Comunicación personal. 